\title{
Intergalactic dust and its photoelectric heating
}

\author{
Akio K. Inoue ${ }^{1}$ and Hideyuki Kamaya ${ }^{2}$ \\ ${ }^{1}$ College of General Education, Osaka Sangyo University, \\ 3-1-1, Nakagaito, Daito, Osaka 574-8530, Japan \\ ${ }^{2}$ Department of Earth and Ocean Sciences, National Defense Academy of Japan, \\ Hashirimizu 1-10-20, Yokosuka, Kanagawa 239-8686, Japan
}

(Received July 31, 2008; Revised October 21, 2008; Accepted October 29, 2008; Online published February 12, 2010)

\begin{abstract}
We have examined dust photoelectric heating in the intergalactic medium (IGM). The heating rate in a typical radiation field of the IGM is represented by $\Gamma_{\mathrm{pe}}=1.2 \times 10^{-34} \mathrm{erg} \mathrm{s}^{-1} \mathrm{~cm}^{-3}$ $\left(\mathcal{D} / 10^{-4}\right)\left(n_{\mathrm{H}} / 10^{-5} \mathrm{~cm}^{-3}\right)^{4 / 3}\left(J_{\mathrm{L}} / 10^{-21} \mathrm{erg} \mathrm{s}^{-1} \mathrm{~cm}^{-2} \mathrm{~Hz}^{-1} \mathrm{sr}^{-1}\right)^{2 / 3}\left(T / 10^{4} \mathrm{~K}\right)^{-1 / 6}$, where $\mathcal{D}$ is the dust-to-gas mass ratio, $n_{\mathrm{H}}$ is the hydrogen number density, $J_{\mathrm{L}}$ is the mean intensity at the hydrogen Lyman limit of the background radiation, and $T$ is the gas temperature, if we assume the new X-ray photoelectric yield model by Weingartner et al. (2006) and the dust size distribution in the Milky Way by Mathis et al. (1977). This heating rate dominates the $\mathrm{HI}$ and HeII photoionization heating rates when the hydrogen number density is less than $\sim 10^{-6} \mathrm{~cm}^{-3}$ if $\mathcal{D}=10^{-4}$ which is $1 \%$ of that in the Milky Way, although the heating rate is a factor of $2-4$ smaller than that with the old yield model by Weingartner and Draine (2001). The grain size distribution is very important. If only large $(\geq 0.1 \mu \mathrm{m})$ grains exist in the IGM, the heating rate is reduced by a factor of $\simeq 5$. Since dust heating is more efficient in a lower density medium relative to the photoionization heating, it may cause an inverted temperature-density relation in the low-density IGM, as suggested by Bolton et al. (2008). Finally, we have found that dust heating is not very important in the mean IGM before the cosmic reionization.
\end{abstract}

Key words: Dust grains, intergalactic medium, photo-electron, photo-ionization.

\section{Introduction}

Dust grains are formed at the end of the stellar life, in the stellar wind of asymptotic giant branch stars (e.g., Ferrarotti and Gail, 2006), in the stellar ejecta of supernovae (e.g., Nozawa et al., 2003; Rho et al., 2008), among others. Some of the grains grow in molecular clouds (e.g., Draine, 1990), others are destroyed by the interstellar shock (e.g., Williams et al., 2006), and some of them may go out from the parent galaxy and reach the intergalactic medium (IGM) (e.g., Aguirre et al., 2001a, b).

The IGM is the medium between galaxies, and it occupies almost the whole volume of the Universe. The mean density of the IGM is as low as $10^{-7}-10^{-4} \mathrm{~cm}^{-3}$. As found by Gunn and Peterson (1965), the IGM is highly ionized after the cosmic reionization epoch (the redshift $z \simeq 6$ 10; Loeb and Barkana, 2001; Fan et al., 2006). Thus, its temperature is $\sim 10^{4} \mathrm{~K}$. The IGM is filled with the ionizing ultra-violet (UV) and X-ray background radiation which is produced by QSOs and galaxies (e.g., Haardt and Madau, 1996).

A significant amount of metals is found in the IGM (e.g., Aguirre et al., 2001c). Multiple supernova explosions (SN) caused by an active star-formation in galaxies can eject the metal elements to the IGM. However, Ferrara et al. (2000) showed that the metal enrichment of the IGM by SN ex-

Copyright (C) The Society of Geomagnetism and Earth, Planetary and Space Sciences (SGEPSS); The Seismological Society of Japan; The Volcanological Society of Japan; The Geodetic Society of Japan; The Japanese Society for Planetary Sciences; TERRAPUB.

doi: $10.5047 /$ eps.2008.10.003 plosions is limited to relatively small regions around starforming galaxies, and an additional physical mechanism is required to explain the observed global enrichment of metals in the IGM. Dust grains expelled from galaxies by the radiation pressure due to stellar light and by the galactic wind due to multiple $\mathrm{SNe}$ may contribute to the metal enrichment in the IGM (e.g., Aguirre et al., 2001a, b). Bianchi and Ferrara (2005) showed that relatively large $(>0.1 \mu \mathrm{m})$ dust grains are not completely destroyed and reach a significant distance $(\mathrm{a}$ few $\times 100 \mathrm{kpc}$ ) although the amount of this intergalactic dust is too small to make a detectable extinction.

Infrared (IR) emission from dust grains in the IGM surrounding edge-on galaxies has been already detected (e.g., Alton et al., 1999; Bendo et al., 2006). Moreover, IR emission from dust in the IGM accumulated from the distant Universe may affect the cosmic far-IR background and the cosmic microwave background (Aguirre and Haiman, 2000). Emission signature from dust even at the epoch of the cosmic reionization may be detectable with a future satellite observing the cosmic microwave background (Elfgren et al., 2007).

Xilouris et al. (2006) found a significant reddening of galaxies behind a giant cloud detected by HI $21 \mathrm{~cm}$ emission in the M81 group (e.g., Yun et al., 1994). Their measurements imply that the dust-to-gas ratio in the M81 group IGM is a factor of 5 larger than that in the Milky Way. Such a large amount of dust in the IGM may be ejected from M82 by its intense starburst activity (Alton et al., 1999).

Dust in the IGM affects results from the precision cos- 
mology. Indeed, high redshift SNe Ia are dimmed by dust in the IGM, conseqently, the observational estimate of the distance to them and cosmological parameters become ambiguous (Goobar et al., 2002). Furthermore, future investigations of the 'equation of state' of the Dark energy will be affected by the extinction of the intergalactic dust even if its amount is too small to affect the conclusion of the presence of the Dark energy (Corasaniti, 2006; Zhang and Corasaniti, 2007).

Dust in the IGM also affects the thermal history of the IGM. In the intracluster medium, dust grains work as a coolant because they emit energy obtained from gas particles collisionally as the thermal IR radiation (Montier and Giard, 2004). Such an emission from some nearby galaxy clusters can be detectable with the current and future satellites for the IR observations (Yamada and Kitayama, 2005). Dust grains in the IGM also work as a heating source via the photoelectric effect (Nath et al., 1999). Inoue and Kamaya $(2003,2004)$ proposed the possibility of obtaining an upper limit of the amount of the intergalactic dust based on the thermal history of the IGM with the dust photoelectric heating.

In this paper, we revisit the effect of the dust photoelectric heating in the IGM. Recently, Weingartner et al. (2006) revised the model of the photoelectric yield of dust grains. They included a few new physical processes; the photon and electron transfer in a grain, the photoelectron emission from the inner shells of the constituent atoms of grains, the secondary electron emission, and the Auger electron emission. These new features reduce the photoelectric yield for moderate energy photons of $\sim 100 \mathrm{eV}$ but enhance the yield for high-energy photons $>1 \mathrm{keV}$. In particular, we explore the effect of this new yield model on the photoelectric heating by the intergalactic dust in this paper.

The rest of this paper consists of four sections; in Section 2, we describe the model of the photoelectric effect. In Section 3, we compare heating rates of the photoelectric effect with those of the photoionization in the IGM. In Section 4, we discuss the implications of the results of Section 3. Final section is devoted to our conclusions.

\section{Dust Photoelectric Effect}

\subsection{Grain charging processes}

To examine the photoelectric effect, we must specify the charge of grains, which is given by the following equation (Spitzer, 1941; Draine and Salpeter, 1979):

$$
\frac{d Z_{\mathrm{d}}}{d t}=\sum_{i} R_{i}+R_{\mathrm{pe}}
$$

where $Z_{\mathrm{d}}$ is the grain charge in the electron charge unit, $R_{i}$ is the collisional charging rate by $i$-th charged particle (hereafter the subscript " $i$ " means " $i$-th charged particle"), and $R_{\mathrm{pe}}$ is the photoelectric charging rate. We consider only protons and electrons as the charged particle.

2.1.1 Collisional charging rate The collisional charging rate by $i$-th charged particle, $R_{i}$, is expressed as (e.g., Draine and Sutin, 1987)

$$
R_{i}=Z_{i} s_{i} n_{i} \int_{0}^{\infty} \sigma_{i}\left(a, Z_{\mathrm{d}}, Z_{i}, v_{i}\right) v_{i} f\left(v_{i}\right) d v_{i},
$$

where $Z_{i}$ is the charge in the electron charge unit, $s_{i}$ is the sticking coefficient, $n_{i}$ is the number density, $v_{i}$ is the velocity, $\sigma_{i}$ is the collisional cross section depending on the grain radius, $a$, both charges, and the velocity, and $f\left(v_{i}\right)$ is the velocity distribution function. If the grain and the charged particle have the charges of the same sign, the kinetic energy of the particle must exceed the grain electric potential for the collision. Otherwise, the collisional cross section is zero. We simply assume $s_{i}$ is always unity.

Now, we introduce the dimensionless cross section, $\tilde{\sigma}_{i}=$ $\sigma_{i} / \pi a^{2}$. If we neglect the "image potential" resulting from the polarization of the grain induced by the Coulomb field of the approaching charged particle (Draine and Sutin, 1987) and we assume the Maxwellian velocity distribution for the particle and the spherical grains, we obtain

$$
\int_{0}^{\infty} \tilde{\sigma}_{i} v_{i} f\left(v_{i}\right) d v_{i}=\left(\frac{8 k_{\mathrm{B}} T}{\pi m_{i}}\right)^{1 / 2} g(x),
$$

and

$$
g(x)=\left\{\begin{array}{ll}
1-x & \text { for } Z_{\mathrm{d}} Z_{i} \leq 0 \\
\exp (-x) & \text { for } Z_{\mathrm{d}} Z_{i}>0
\end{array},\right.
$$

where $k_{\mathrm{B}}$ is the Boltzmann's constant, $T$ is the gas temperature, $m_{i}$ is the particle mass, and $x=e^{2} Z_{\mathrm{d}} Z_{i} / a k_{\mathrm{B}} T$ (Spitzer, 1941).

In fact, the "image potential" works to enhance the collisional cross section (Draine and Sutin, 1987). Although the effect becomes the most important for grains with an around neutral charge, it quickly declines for highly charged grains. Indeed, for the charge ratio of $Z_{\mathrm{d}} / Z_{i}<-3$, which is satisfied in our case, as found below, the increment factor for the cross section by the effect of the "image potential" is less than 1.5 (Draine and Sutin, 1987). Therefore, we neglect the "image potential" in this paper.

2.1.2 Photoelectric charging rate The photoelectric charging rate is given by (e.g., Draine, 1978)

$$
R_{\mathrm{pe}}=\pi a^{2} \int_{0}^{\infty} Q_{v}(a) Y_{v}\left(a, Z_{\mathrm{d}}\right) \frac{4 \pi J_{v}}{h v} d v,
$$

where $Q_{v}$ is the absorption coefficient of grains at the frequency $v, Y_{v}$ is the photoelectric yield, $J_{v}$ is the mean intensity of the incident radiation, and $h$ is the Plank constant. For $Q_{v}$, we adopt the values of "graphite" and "UV smoothed astronomical silicate" by Draine (2003). If the photon energy is smaller than the threshold energy of the photoelectric emission, e.g., the ionization potential or the work function, the yield $Y_{v}=0$.

We adopt a sophisticated model of the photoelectric yield by Weingartner and Draine (2001) and Weingartner et al. (2006) in this paper. The model of Weingartner and Draine (2001) (hereafter the WD01 model) takes into account the primary photoelectron emission from the band structure of grains, a small-size particle effect, and the energy distribution of the photoelectron. On the other hand, Weingartner et al. (2006) (hereafter the $\mathrm{W}+06$ model) add the primary photoelectron emission from inner shells of the constituent atoms of grains, the Auger electron emission, and the secondary electron emission produced by primary electrons and Auger electrons. The transfer of photons absorbed and electrons emitted in a grain is also taken into account. For 


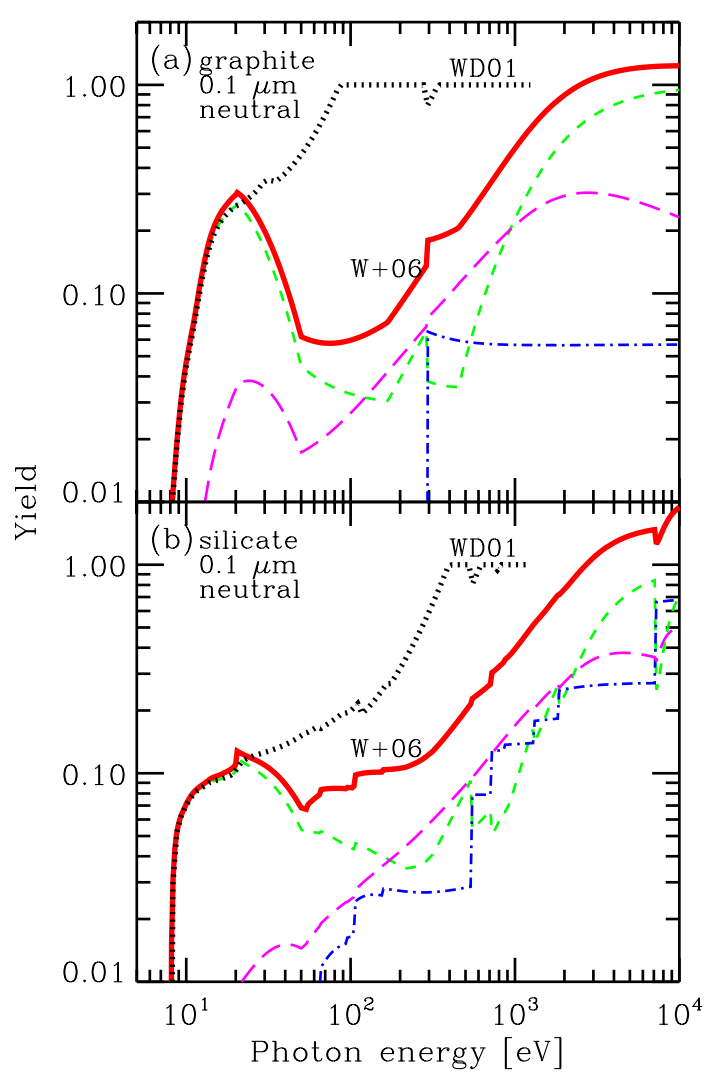

Fig. 1. Photoelectric yield models of $0.1-\mu \mathrm{m}$ neutral (a) graphite and (b) silicate grains. The dotted lines are the WD01 model (Weingartner and Draine, 2001) and the solid lines are the $\mathrm{W}+06$ model (Weingartner et al., 2006). The $\mathrm{W}+06$ model consists of three processes: the primary photoelectron emission (short dashed line), the secondary electron emission (long dashed line), and the Auger electron emission (dot-dashed line).

the detailed procedure of the yield calculations, the reader is referred to the original papers of Weingartner and Draine (2001) and Weingartner et al. (2006). Figure 1 shows comparisons between the WD01 and W+06 models. The reduction of the $\mathrm{W}+06$ yield around $100 \mathrm{eV}$ is due to the effect of the photon/electron transfer in a grain. The $\mathrm{W}+06$ yield exceeds unity for some cases because of the Auger and secondary electrons.

We have to note that there is still a large uncertainty of photoelectric yield models because of our insufficient understandings of the nature of the small-size particle effect as well as the lack of experiments. Abbas et al. (2006) reported measurements of the yield of individual grains of silica, olivine, and graphite with $0.09-5 \mu \mathrm{m}$ radii for $8-$ $10 \mathrm{eV}$ photons. Their measurements indeed show larger yields than those of the bulk materials. However, the measurements do not agree with the yield enhancement factors adopted in the WD01 and W+06 models accounting for the small-size particle effect qualitatively as well as quantitatively. Clearly, we need more experiments and theoretical investigations of the photoelectric yield in future.

2.1.3 Equilibrium charge We need to specify the radiation filed incident on grains in the IGM: the cosmic background radiation. We assume a simple description of the radiation. The intensity of the radiation at the Lyman limit is estimated from observations of the proxim- ity effect and the Lyman $\alpha$ forest opacity (e.g., Scott et al., 2000). A typical value of the intensity at the Lyman limit is $J_{\mathrm{L}}=1 \times 10^{-21} \mathrm{erg} \mathrm{s}^{-1} \mathrm{~cm}^{-2} \mathrm{~Hz}^{-1} \mathrm{sr}^{-1}$ (e.g., Scott et al., 2000). We simply assume a power-law as the spectral shape: $J_{v} \propto v^{-p}$. A typical value of $p$ is unity (e.g., Haardt and Madau, 1996). With such a radiation field, the grains in the IGM are positively charged.

A typical charging time-scale is very short. For example, the collisional charging rate of the electron is $R_{\mathrm{e}} \sim$ $5.6 \times 10^{-6} \mathrm{~s}^{-1}$ for $n_{\mathrm{e}}=10^{-5} \mathrm{~cm}^{-3}, T=10^{4} \mathrm{~K}$, $a=0.1 \mu \mathrm{m}$, and $Z_{\mathrm{d}}=1700$, which is the equilibrium charge of graphite or silicate grains for these parameters and $J_{v}=10^{-21}\left(v / v_{\mathrm{L}}\right)^{-1} \mathrm{erg} \mathrm{s}^{-1} \mathrm{~cm}^{-2} \mathrm{~Hz}^{-1} \mathrm{sr}^{-1}$. Thus, the typical charging time-scale is $t \sim 1 / R_{\mathrm{e}} \sim 6 \times 10^{-3}$ year. Therefore, the grain charge can be in equilibrium. We set $d Z_{\mathrm{d}} / d t=0$ in Eq. (1) and obtain the equilibrium charge of the IGM grains.

\subsection{Heating rates}

2.2.1 Heating rate per a grain The net heating rate per a grain with the radius $a$ is expressed as (e.g., Weingartner and Draine, 2001)

$$
\gamma(a)=R_{\mathrm{pe}} \mathcal{E}_{\mathrm{pe}}(a)-\left|R_{\mathrm{e}}\right| \mathcal{E}_{\mathrm{e}}(T),
$$

where $\mathcal{E}_{\mathrm{pe}}(a)$ is the mean kinetic energy of photoelectrons from a grain with radius $a$, and $\mathcal{E}_{\mathrm{e}}(T)$ is that of electrons colliding with the grain. The second term accounts for the cooling by the electron capture. If we assume the Maxwellian velocity distribution for the electrons, $\mathcal{E}_{\mathrm{e}}(T)=$ $k_{\mathrm{B}} T(2+\phi) /(1+\phi)$, where $\phi=Z_{\mathrm{d}} e^{2} / a k_{\mathrm{B}} T$ (for $Z_{\mathrm{d}}>0$; Draine, 1978). We note that $\mathcal{E}_{\mathrm{e}}$ is $\sim 1 \%$ of $\mathcal{E}_{\mathrm{pe}}$ in the current setting.

The mean energy of the photoelectrons is given by

$$
\mathcal{E}_{\mathrm{pe}}(a)=\frac{\pi a^{2}}{R_{\mathrm{pe}}} \int_{0}^{\nu_{\max }} Q_{v}(a) Y E_{v}\left(a, Z_{\mathrm{d}}\right) \frac{4 \pi J_{v}}{h v} d v,
$$

and

$$
Y E_{v}\left(a, Z_{\mathrm{d}}\right)=\sum_{k} Y_{v}^{k}\left(a, Z_{\mathrm{d}}\right)\left\langle E_{\mathrm{e}}\right\rangle_{v}^{k}\left(a, Z_{\mathrm{d}}\right)
$$

where $Y_{v}^{k}$ is the photoelectric yield of $k$-th emission process, e.g., primary electrons from the band structure, Auger electron, etc., and $\left\langle E_{\mathrm{e}}\right\rangle_{v}^{k}$ is the mean energy of electrons emitted by $k$-th process with the absorbed photon energy $h v$. The estimation of $\left\langle E_{\mathrm{e}}\right\rangle_{v}^{k}$ is based on the assumed energy distribution of the electrons. Following Weingartner et al. (2006), we adopt a parabolic function for the primary and the auger electrons and a function introduced by Draine and Salpeter (1979) for the secondary electrons, which were derived to fit some experimental results.

In the IGM, the grains are positively charged. Therefore, the proton collisional charging rate is negligible. Thus, the photoelectric charging rate balances with the electron collisional charging rate: $R_{\mathrm{pe}}+R_{\mathrm{e}}=0$. In this case, Eq. (6) is reduced to

$$
\begin{aligned}
\gamma(a) & =\left|R_{\mathrm{e}}\right|\left(\mathcal{E}_{\mathrm{pe}}-\mathcal{E}_{\mathrm{e}}\right) \\
& \approx \pi a^{2} n_{\mathrm{e}}\left(\frac{8 k_{\mathrm{B}} T}{\pi m_{\mathrm{e}}}\right)^{1 / 2}\left(\frac{e V_{\mathrm{d}}}{k_{\mathrm{B}} T}\right) \mathcal{E}_{\mathrm{pe}},
\end{aligned}
$$


where we have used Eqs. (2-4) for $R_{\mathrm{e}}$ and $V_{\mathrm{d}}=Z_{\mathrm{d}} e / a$ is the grain electric potential $\left(e V_{\mathrm{d}} / k_{\mathrm{B}} T \gg 1\right.$ and $\mathcal{E}_{\mathrm{pe}} \gg \mathcal{E}_{\mathrm{e}}$ for the IGM). As found later in Fig. 2, the electric potential depends weakly on the grain size in the $\mathrm{W}+06$ yield case. We confirmed that the mean energy of photoelectrons also depends weakly on the grain size. As a result, the heating rate per grain is roughly proportional to the square of the size, which is shown later in Fig. 3.

2.2.2 Total photoelectric heating rate To estimate the total photoelectric heating rate per unit volume, we need to specify the amount and the size distribution of dust grains. A power law type distribution for grain size is familiar in the interstellar medium of the Milky Way since the classical work by Mathis et al. (1977, hereafter MRN). The power law is expected to be achieved as a result coagulation, shattering, and sputtering processes (e.g., Jones et al., 1996). Here we express the power law distribution as $n(a)=A a^{-q}$, where $n(a) d a$ is the number density of grains with the radius between $a$ and $a+d a$. For the MRN distribution, $q=3.5$ (see Table 1 ). The normalization $A$ is determined from the total dust mass density $\rho_{\mathrm{d}}=\int_{a_{\min }}^{a_{\max }} m(a) n(a) d a$, where $m(a)=(4 \pi / 3) \varrho a^{3}$ is the mass of grains with the radius $a, \varrho\left(\simeq 3 \mathrm{~g} \mathrm{~cm}^{-3}\right)$ is the grain material density, and $a_{\min }$ and $a_{\max }$ are the minimum and maximum radius, respectively. The dust mass density $\rho_{d}$ is given by $\rho_{\mathrm{d}}=m_{\mathrm{p}} n_{\mathrm{H}} \mathcal{D}$, where $m_{\mathrm{p}}$ is the proton mass, $n_{\mathrm{H}}$ is the hydrogen number density, and $\mathcal{D}$ is the dust-to-gas mass ratio. We assume $\mathcal{D}=10^{-4}$, which is about two orders of magnitude smaller than that in the Milky Way's ISM. Then, the total photoelectric heating rate is

$$
\Gamma_{\mathrm{pe}}=\int_{a_{\min }}^{a_{\max }} \gamma(a) n(a) d a .
$$

Let us consider a typical size for the total heating rate. Using the grain number density $n_{\mathrm{d}}=\int_{a_{\min }}^{a_{\max }} n(a) d a$, we can define a mean heating rate per grain as $\langle\gamma\rangle \equiv$ $\int_{a_{\min }}^{a_{\max }} \gamma(a) n(a) d a / n_{\mathrm{d}}$ and a mean mass per grain as $\left\langle m_{\mathrm{d}}\right\rangle \equiv$ $\rho_{\mathrm{d}} / n_{\mathrm{d}}=\int_{a_{\min }}^{a_{\max }} m(a) n(a) d a / n_{\mathrm{d}}$. Then, the total heating rate is reduced to $\Gamma_{\mathrm{pe}}=\langle\gamma\rangle n_{\mathrm{d}}=\langle\gamma\rangle \rho_{\mathrm{d}} /\left\langle m_{\mathrm{d}}\right\rangle$. The heating rate per a grain can be approximated to $\gamma(a) \approx \gamma_{0} a^{2}$ as seen in Section 2.2.1 (see also Fig. 3 and Section 3.1.2), where $\gamma_{0}$ is a normalization. The grain mass is $m(a)=(4 \pi / 3) \varrho a^{3}$. Then, we obtain

$$
\Gamma_{\mathrm{pe}} \approx \frac{3 \rho_{\mathrm{d}} \gamma_{0}}{4 \pi \varrho\langle a\rangle}
$$

where a typical size $\langle a\rangle$ is given by

$$
\langle a\rangle=\frac{\int_{a_{\min }}^{a_{\max }} a^{3} n(a) d a}{\int_{a_{\min }}^{a_{\max }} a^{2} n(a) d a} .
$$

Note that a larger typical size results in a smaller total heating rate because of a smaller number density of grains for a fixed dust mass.

2.2.3 Photoionization heating rates For comparison with the photoelectric heating rate by grains, we estimate the photoionization heating rates of hydrogen and helium. The net HI photoionization heating rate is

$$
\Gamma_{\mathrm{pi}}^{\mathrm{HI}}=n_{\mathrm{HI}} R_{\mathrm{pi}}^{\mathrm{HI}} \mathcal{E}_{\mathrm{pi}}^{\mathrm{HI}}-n_{\mathrm{HII}} R_{\mathrm{re}}^{\mathrm{HI}} \mathcal{E}_{\mathrm{gas}},
$$

where $R_{\mathrm{pi}}^{\mathrm{HI}}=\int_{\nu_{\mathrm{L}}^{\mathrm{HI}}}^{\infty} \sigma_{\nu}^{\mathrm{HI}} 4 \pi J_{\nu} / h \nu d \nu$ is the HI photoionization rate, $R_{\mathrm{re}}^{\mathrm{HI}}=n_{\mathrm{e}} \alpha_{\mathrm{A}}^{\mathrm{HI}}(T)$ is the $\mathrm{HI}$ recombination rate, $\mathcal{E}_{\mathrm{pi}}^{\mathrm{HI}}=$ $\left(1 / R_{\mathrm{pi}}^{\mathrm{HI}}\right) \int_{\nu_{\mathrm{L}}^{\mathrm{HI}}}^{\infty} \sigma_{v}^{\mathrm{HI}} 4 \pi J_{v} / h v\left(h v-h v_{\mathrm{L}}^{\mathrm{HI}}\right) d v$ is the mean kinetic energy of the HI photoionized electrons, $\sigma_{v}^{\mathrm{HI}}$ is the $\mathrm{HI}$ photoionization cross section, $v_{\mathrm{L}}^{\mathrm{HI}}$ is the HI Lyman limit frequency, $n_{\mathrm{HI}}, n_{\mathrm{HII}}$, and $n_{\mathrm{e}}$ are the neutral hydrogen, ionized hydrogen, and electron number densities, respectively, $\alpha_{\mathrm{A}}^{\mathrm{HI}}(T)$ is the Case A HI recombination coefficient for the gas temperature $T$ (Osterbrock and Ferland, 2006), and $\mathcal{E}_{\text {gas }}$ is the mean kinetic energy lost from the gas per one recombination. If we assume that $J_{v} \propto v^{-p}$ and $\sigma_{v}^{\mathrm{HI}} \propto v^{-3}$, we obtain $\mathcal{E}_{\mathrm{pi}}^{\mathrm{HI}}=h v_{\mathrm{L}}^{\mathrm{HI}} /(p+2)$. If we take into account the gas cooling by free-free emission, $\mathcal{E}_{\text {gas }} \approx k_{\mathrm{B}} T$ for the Case $\mathrm{A}$ and $T=10^{4} \mathrm{~K}$ (Osterbrock and Ferland, 2006). If we assume the ionization equilibrium, $n_{\mathrm{HI}} R_{\mathrm{pi}}^{\mathrm{HI}}=n_{\mathrm{HII}} R_{\mathrm{re}}^{\mathrm{HI}}$, we obtain

$$
\Gamma_{\mathrm{pi}}^{\mathrm{HI}}=n_{\mathrm{H}}^{2} \alpha_{\mathrm{A}}^{\mathrm{HI}}(T)\left(\mathcal{E}_{\mathrm{pi}}^{\mathrm{HI}}-\mathcal{E}_{\mathrm{gas}}\right),
$$

where we have assumed $n_{\mathrm{HII}}=n_{\mathrm{e}}=n_{\mathrm{H}}$ with $n_{\mathrm{H}}$ being the hydrogen number density, that is, the neutral fraction is assumed to be very small. The net HeII photoionization heating rate is likewise

$$
\Gamma_{\mathrm{pi}}^{\mathrm{HeII}}=n_{\mathrm{He}} n_{\mathrm{H}} \alpha_{\mathrm{A}}^{\mathrm{HeII}}(T)\left(\mathcal{E}_{\mathrm{pi}}^{\mathrm{HeII}}-\mathcal{E}_{\mathrm{gas}}\right)
$$

where $n_{\mathrm{He}}$ is the helium number density, $\alpha_{\mathrm{A}}^{\mathrm{HeII}}(T)$ is the HeII recombination rate, and $\mathcal{E}_{\mathrm{pi}}^{\mathrm{HeII}}$ is the mean kinetic energy of the HeII photoionized electrons. We assume $n_{\mathrm{He}} / n_{\mathrm{H}}=0.1$.

\section{Results}

\subsection{Comparison between the two yield models}

We compare the grain charge and heating rates with the WD01 and W+06 models quantitatively in the IGM environment. Weingartner et al. (2006) showed the grain charges in the QSO environments in a similar situation and similar radiation field as those reported in this paper. However, they did not show the heating rates in the environment.

3.1.1 Electric potential In Fig. 2, we compare the electric potentials of the $\mathrm{W}+06$ model (solid lines) with those of the WD01 model (dashed lines). We show two cases of the spectrum of the radiation field; one has a hard spectrum as a background radiation dominated by QSOs, which is the case with the spectral index $p=1$, and the other has a soft spectrum with $p=5$ for a comparison. Other assumed quantities are appropriate for the IGM at the redshift $z \sim 3$ and are shown in the panels. The radiation fields assumed here correspond to the ionization parameter $U \equiv n_{\text {ion }} / n_{\mathrm{H}}$, which is the number density ratio of ionizing photons and hydrogen nucleus, of 6.3 for $p=1$ and of 1.3 for $p=5$. Weingartner et al. (2006) showed electric potentials in their figures 6 and 7 , with $U=0.1-100$. We find that our calculations are quantitatively well-matched with theirs.

We find in Fig. 2 that for the hard spectrum case, the grain electric potentials with the $\mathrm{W}+06$ yield model are much smaller than those with the WD01 model, especially for larger grain sizes. On the other hand, for the soft spectrum case, the difference is very small, less than $4 \%$. This 


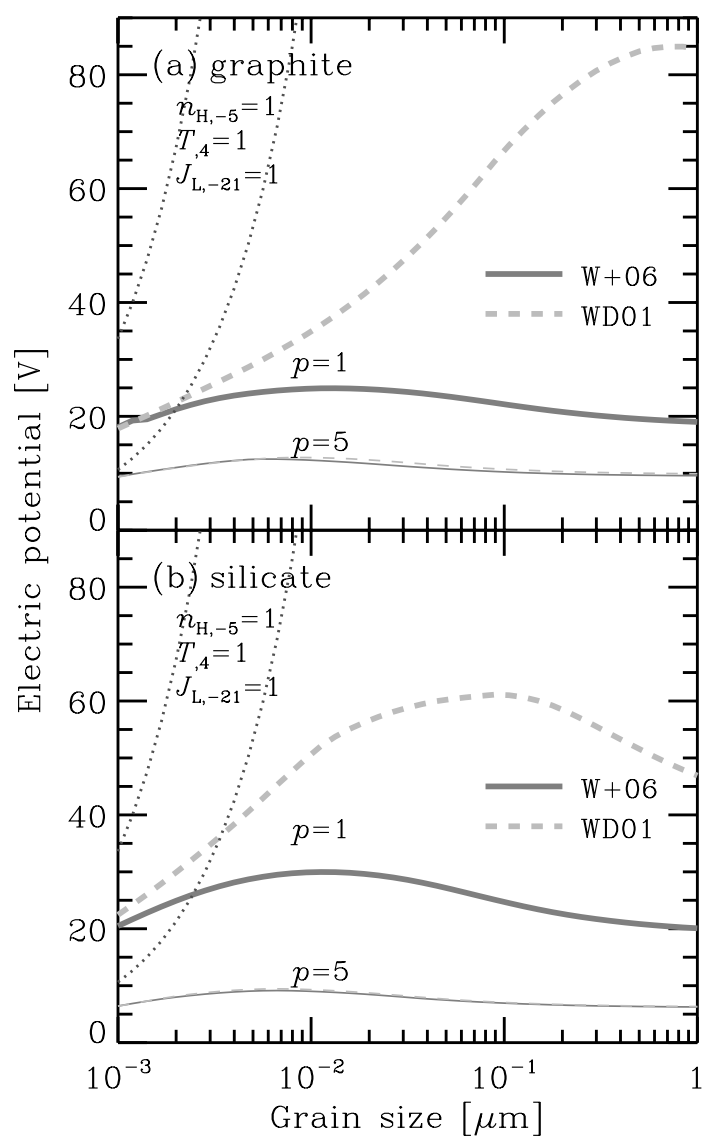

Fig. 2. Equilibrium electric potential as a function of grain size: (a) graphite and (b) silicate. The solid lines are the $\mathrm{W}+06$ model and the dashed lines are the WD01 model. The thick lines are the case with the spectral index of the radiation field $p=1$ and the thin lines are the case with $p=5$. Other assumed quantities are noted in the panels as the hydrogen density $n_{\mathrm{H},-5}=n_{\mathrm{H}} / 10^{-5} \mathrm{~cm}^{-3}$, the gas temperature $T_{, 4}=T / 10^{4} \mathrm{~K}$, and the radiation intensity $J_{\mathrm{L},-21}=J_{\mathrm{L}} / 10^{-21} \mathrm{erg} \mathrm{s}^{-1} \mathrm{~cm}^{-2} \mathrm{~Hz}^{-1} \mathrm{sr}^{-1}$. The dotted lines show the critical electric potential where the grain destruction occurs by the Coulomb explosion; the upper lines are the case with the tensile strength of $10^{11} \mathrm{dyn}^{-2}$, and the lower lines are the case with $10^{10} \mathrm{dyn}^{-2}$.

is because the main difference between the $\mathrm{W}+06$ yield and the WD01 yield is found in the primary photoelectron yield at $\sim 100 \mathrm{eV}$ due to the photon/electron transfer in a grain as shown in Fig. 1. In the soft spectrum case, since there are not many photons around the energy, we do not find a significant difference between the two yield models. For smaller grain sizes, the yield reduction by the photon/electron transfer is small as found in figures 4 and 5 of Weingartner et al. (2006). Thus, we do not find a significant difference in the electric potentials for smaller grain sizes in Fig. 2 either.

The electrostatic stress on a grain may cause the grain destruction by the Coulomb explosion (e.g., Draine and Salpeter, 1979). The critical electric potential is $V_{\max }=$ $1063 \mathrm{~V}\left(S_{\mathrm{d}} / 10^{10} \mathrm{dyn} \mathrm{cm}^{-2}\right)^{1 / 2}(a / 0.1 \mu \mathrm{m})$, where $S_{\mathrm{d}}$ is the tensile strength of grains, which is very uncertain. Perfect crystal structure may have $S_{\mathrm{d}} \sim 10^{11}$ dyn $\mathrm{cm}^{-2}$ (Draine and Salpeter, 1979), but imperfections would reduce the strength as $S_{\mathrm{d}} \sim 10^{10}$ dyn $\mathrm{cm}^{-2}$ (Fruchter et al., 2001). Following Weingartner et al. (2006), we show two cases of the critical potential with $S_{\mathrm{d}} \sim 10^{10}$ and $10^{11}$ dyn

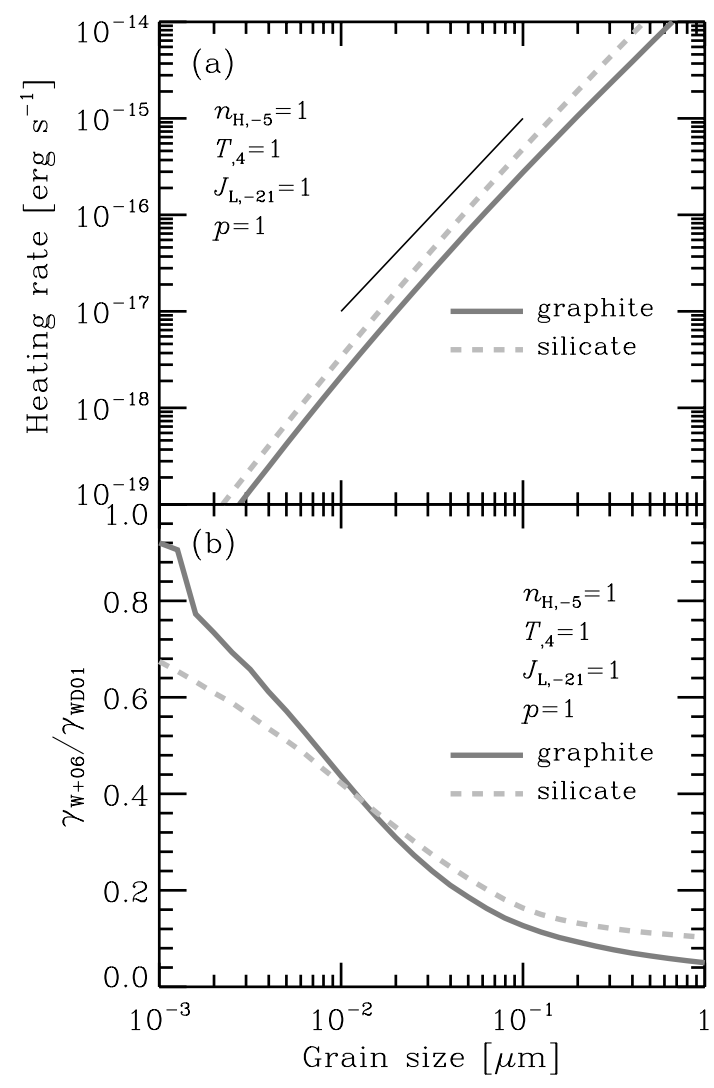

Fig. 3. Photoelectric heating rate per grain as a function of grain size: (a) heating rate with the $\mathrm{W}+06$ yield model and (b) ratio of the heating rate with the W+06 yield model to that with the WD01 yield model. The solid lines are the graphite case and the dashed lines are the silicate case. The assumed quantities are noted in the panels as the hydrogen density $n_{\mathrm{H},-5}=n_{\mathrm{H}} / 10^{-5} \mathrm{~cm}^{-3}$, the gas temperature $T_{, 4}=T / 10^{4} \mathrm{~K}$, the radiation intensity $J_{\mathrm{L},-21}=J_{\mathrm{L}} / 10^{-21} \mathrm{erg} \mathrm{s}^{-1} \mathrm{~cm}^{-2} \mathrm{~Hz}^{-1} \mathrm{sr}^{-1}$, and the spectral index of the radiation field $p=1$. The thin solid line in the panel (a) shows the slope proportional to the square of size.

$\mathrm{cm}^{-2}$ in Fig. 2 as the dotted lines. The critical potential by the ion field emission is similar to the case with $S_{\mathrm{d}} \sim 10^{11} \mathrm{dyn} \mathrm{cm}^{-2}$ (Draine and Salpeter, 1979). We find that grains smaller than 20-30 $\AA$ in the hard radiation field may be destroyed by the Coulomb explosion. As such, there may be no very small grains in the IGM.

3.1.2 Photoelectric heating rate Figure 3 shows the photoelectric heating rate per grain in a typical $z \sim 3 \mathrm{IGM}$ environment with hard radiation; graphite grains are shown by solid lines and silicate grains are shown by the dashed lines. In panel (a), we show the absolute value of the heating rate for the $W+06$ yield model. As expected in Eq. (9), the heating rate is nicely proportional to $a^{2}$, square of the size. However, the slope becomes gradually steep for a small $(<100 \AA ̊)$ grain size.

In panel (b), we compare the two heating rates with the W+06 model and the WD01 model. We find that the heating rate with the $\mathrm{W}+06$ model is much smaller than that with the WD01 model: a factor of 10 smaller for the largest grain size. This is because (1) reduction of the grain electric potential and (2) reduction of the mean energy of the photoelectron in the W+06 model. As found in Eq. (9), the heating rate per grain is proportional to the product of the potential and the mean photoelectron energy. As seen in 


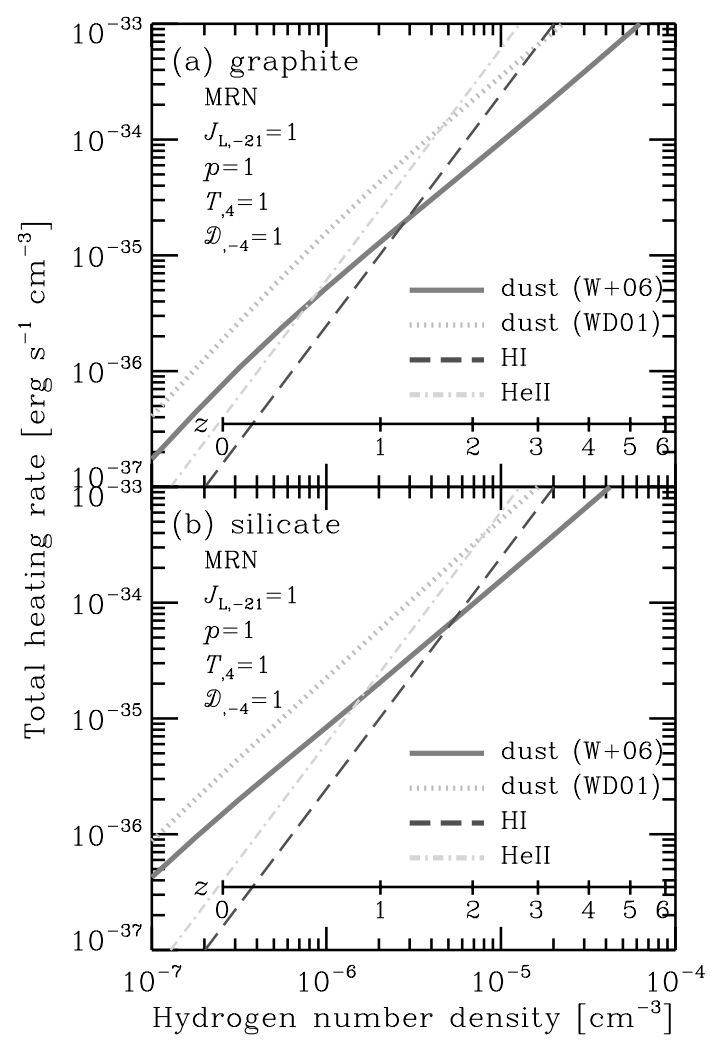

Fig. 4. Photoelectric heating rates as a function of hydrogen number density: (a) graphite and (b) silicate. The solid lines are the $\mathrm{W}+06$ model and the dotted lines are the WD01 model. The assumed grain size distribution is the so-called MRN distribution (Mathis et al., 1977). Other assumed quantities are noted in the panels as the gas temperature $T_{, 4}=T / 10^{4} \mathrm{~K}$, the radiation intensity $J_{\mathrm{L},-21}=J_{\mathrm{L}} / 10^{-21} \mathrm{erg} \mathrm{s}^{-1} \mathrm{~cm}^{-2} \mathrm{~Hz}^{-1} \mathrm{sr}^{-1}$, the spectral index of the radiation field $p$, and the dust-to-gas mass ratio $\mathcal{D}_{-4}=\mathcal{D} / 10^{-4}$. The dashed lines are the HI photoionization heating rate and the dot-dashed lines are the HeII photoionization heating rate assuming ionization equilibrium. We also show the redshift at which the number density on the horizontal axis corresponds to the mean density of the Universe.

Fig. 2, smaller potential is expected with the $\mathrm{W}+06$ model because of the reduction of the yield at $\sim 100 \mathrm{eV}$. The yield reduction also causes the reduction of the mean energy of the photoelectron, as expected in Eq. (8). Therefore, we have up to about a factor of 10 reduction of the heating rate with the $\mathrm{W}+06$ model.

Figure 4 shows a comparison of the total heating rates by the W+06 model (solid lines) and by the WD01 model (dotted lines). The horizontal axis is the assumed hydrogen number density. We also show the redshift at which the number density on the horizontal axis corresponds to the mean density of the Universe. We have assumed the MRN grain size distribution (see Table 1). We find that the total heating rate with the $\mathrm{W}+06$ yield is a factor of 2-4 smaller than that with the WD01 yield.

For a comparison, we also show the HI and HeII photoionization heating rates in Fig. 4. We have assumed the ionization equilibrium for these. When we assume the dustto-gas ratio in the IGM is $1 \%$ of that in the Milky Way (i.e., $\mathcal{D}=10^{-4}$ ), the dust photoelectric heating dominates the HI and HeII photoionization heatings if the hydrogen number density is less than $10^{-6}-10^{-5} \mathrm{~cm}^{-3}$, which corresponds
Table 1. Possible size distributions of the intergalactic dust.

\begin{tabular}{|c|c|}
\hline MRN & Mathis et al. (1977) \\
\hline \multicolumn{2}{|c|}{ Single power law ${ }^{\mathrm{a}}$} \\
\hline$q$ & 3.5 \\
\hline$a_{\min }$ & $50 \AA$ \\
\hline$a_{\max }$ & $0.25 \mu \mathrm{m}$ \\
\hline$\langle a\rangle$ & $350 \AA$ \\
\hline BF05 & Bianchi and Ferrara (2005) \\
\hline \multicolumn{2}{|c|}{ Single power law ${ }^{\mathrm{a}}$} \\
\hline$q$ & 3.5 \\
\hline$a_{\min }$ & $0.1 \mu \mathrm{m}$ \\
\hline$a_{\max }$ & $0.25 \mu \mathrm{m}$ \\
\hline$\langle a\rangle$ & $0.16 \mu \mathrm{m}$ \\
\hline N03 & Nozawa et al. (2003) \\
\hline \multicolumn{2}{|c|}{ Double power law ${ }^{\mathrm{b}}$} \\
\hline$q_{1}\left(a \leq a_{\mathrm{c}}\right)$ & 2.5 \\
\hline$q_{2}\left(a>a_{\mathrm{c}}\right)$ & 3.5 \\
\hline$a_{\min }$ & $2 \AA$ \\
\hline$a_{\max }$ & $0.3 \mu \mathrm{m}$ \\
\hline$a_{\mathrm{c}}$ & $0.01 \mu \mathrm{m}$ \\
\hline$\langle a\rangle$ & $290 \AA$ \\
\hline N07 & Nozawa et al. (2007) \\
\hline \multicolumn{2}{|c|}{ Double power law ${ }^{b}$} \\
\hline$q_{1}\left(a \leq a_{\mathrm{c}}\right)$ & 1.0 \\
\hline$q_{2}\left(a>a_{\mathrm{c}}\right)$ & 2.5 \\
\hline$a_{\min }$ & $10 \AA$ \\
\hline$a_{\max }$ & $0.3 \mu \mathrm{m}$ \\
\hline$a_{\mathrm{c}}$ & $0.01 \mu \mathrm{m}$ \\
\hline$\langle a\rangle$ & $0.12 \mu \mathrm{m}$ \\
\hline SG & - \\
\hline \multicolumn{2}{|c|}{ Single power law ${ }^{\mathrm{a}}$} \\
\hline$q$ & 3.5 \\
\hline$a_{\min }$ & $50 \AA$ \\
\hline$a_{\max }$ & $0.025 \mu \mathrm{m}$ \\
\hline$\langle a\rangle$ & $110 \AA$ \\
\hline
\end{tabular}

${ }^{\text {a }}$ The grain size distribution $n(a) \propto a^{-q}$.

${ }^{\mathrm{b}}$ The grain size distribution $n(a) \propto a^{-q_{1}}$ for $a \leq a_{\mathrm{c}}$ and $\propto a^{-q_{2}}$ for $a>a_{\mathrm{c}}$.

to the redshift $z \sim 1-2$. We note that the dust heating is the most important mechanism in the IGM at $z=0$ even with the $\mathrm{W}+06$ yield model if the IGM has dust with $1 \%$ dust-to-gas ratio of the Milky Way and with the MRN size distribution.

\subsection{Effect of the grain size distribution}

The size distribution of the intergalactic dust grains should be important for the photoelectric heating rate via the typical size defined by Eq. (12). However, it is quite uncertain. Thus, we examine several possibilities of the size distribution in this section. Table 1 is a summary of the size distribution considered here.

The grain size distribution in the Milky Way has been approximated to be a power-law since Mathis et al. (1977) suggested $n(a) \propto a^{-q}$ with $q=3.5$. This MRN distribution is a reference case and is already adopted in Fig. 4. During the grain transport from galaxies to the IGM, there may be size-filtering mechanisms. For example, Ferrara et al. (1991) showed that sputtering in the hot gas filling the galactic halo efficiently destroys grains smaller than $\sim 0.1 \mu \mathrm{m}$. Bianchi and Ferrara (2005) also showed that only 
grains larger than $\sim 0.1 \mu \mathrm{m}$ reach a significant distance (a few $\times 100 \mathrm{kpc}$ ) from the parent galaxies by calculating the grain ejection by the radiation pressure and the grain destruction by the sputtering simultaneously. Here, we consider a simple size distribution of the MRN with $\geq 0.1 \mu \mathrm{m}$ grains as the BF05 model.

In the early Universe, the dominant source of dust grains is different from that in the current Milky Way. Although asymptotic giant branch stars are considered to be the main dust source in the Milky Way (e.g., Dwek, 1998), there is not enough time for stars to evolve to the phase in the early Universe at the redshift $z>6$. However, plenty of dust is found in QSOs at $z>6$ (Bertoldi et al., 2003). SNe is the candidate of the dust source in the early Universe (e.g., Nozawa et al., 2003), and the observed extinction curve of dust associated with the QSO is compatible with those expected from the grains produced by $\mathrm{SNe}$ (Maiolino et al., 2004; Hirashita et al., 2005, 2008). Thus, we consider the size distribution expected from the SNe dust production model by Nozawa et al. (2003) as the N03 model. In addition, we adopt the size distribution expected by Nozawa et al. (2007), who explored the effect of the dust destruction by the reverse shock in the SN remnant, as the N07 model.

Finally, we adopt a hypothetical size distribution consisting of only small grains as a comparison case; the MRN distribution with the maximum size of $250 \AA$ as the SG (small grain) model.

Figure 5 shows a comparison of total heating rates with the five size distributions considered here. All of the cases are assumed to be the $\mathrm{W}+06$ yield model and have physical conditions appropriate for the IGM. The case of the BF05 model (triple-dot-dashed line) is a factor of $\simeq 5$ smaller than that of the MRN model (thick solid line). This reduction factor is simply accounted for by the ratio of the typical sizes of the two models: $0.16 \mu \mathrm{m}$ for the BF05 model and $350 \AA$ for the MRN model (see Table 1). The same is true for the N07 model (dotted line) and the SG model (thin solid line). The result of the N03 model (dashed line) coincides with that of the MRN model because their typical sizes are similar. In any case, we have a smaller number of grains for a larger typical size if the total dust mass is fixed. Then, the heating rate is reduced. We note that the dust photoelectric heating is still a dominant or important mechanism relative to the $\mathrm{HI}$ and HeII photoionization heatings in the $z=0$ IGM even with the BF05 model if the dust-to-gas ratio in the IGM is $1 \%$ of that in the Milky Way.

\subsection{A simple formula of the dust photoelectric heating rate}

Figure 6 shows the effect of different settings of the calculation on the dust photoelectric heating rate: (a) various intensities of the background radiation and (b) various temperatures of the gas. The $\mathrm{W}+06$ yield model and the MRN size distribution are assumed. We also assume that the dust consists of a mixture of graphite and silicate with the mass ratio of 1:1. The spectral index of the background radiation is always set at unity. In the weakest intensity case (squares in the panel (a)), the equilibrium charges for smallest grains $(<0.01 \mu \mathrm{m})$ are less than 3 in the electric charge unit for the hydrogen density $n_{\mathrm{H}}>2 \times 10^{-5} \mathrm{~cm}^{-3}$. In these cases, the effect of the "image potential" (Draine and Sutin, 1987) is

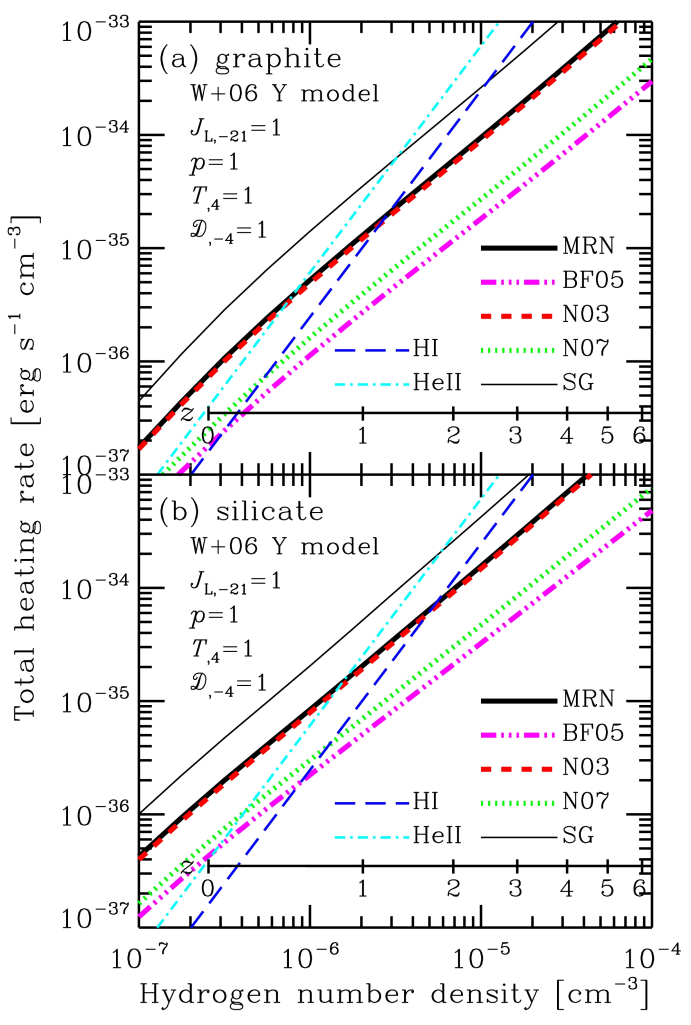

Fig. 5. Same as Fig. 4 but for various size distribution functions with the W+06 yield model: (a) graphite and (b) silicate. The thick solid lines are the MRN case. The short-dashed lines are the size distribution expected from the grain formation model in supernova ejecta by Nozawa et al. (2003). The dotted lines are the size distribution expected after the grain destruction by the reverse shock in the supernova remnant by Nozawa et al. (2007). The triple-dot-dashed lines are the MRN but only of a size larger than $0.1 \mu \mathrm{m}$ because of a filtering effect in the transfer of grains from galaxies to the IGM, as suggested by Bianchi and Ferrara (2005). The thin solid lines are the MRN but only size smaller than $250 \AA$ as a comparison. The dashed and dot-dashed lines are HI and HeII heating rates.

not negligible, and, consequently, the current calculations are no longer valid. We note that all the cases shown in Fig. 6 have an equilibrium charge much larger than 3 for all grains in the size distribution.

The resultant heating rates are well expressed as

$$
\begin{aligned}
\Gamma_{\mathrm{pe}}= & 1.2 \times 10^{-34} \mathrm{erg} \mathrm{s}^{-1} \mathrm{~cm}^{-3} \\
& \times\left(\frac{\mathcal{D}}{10^{-4}}\right)\left(\frac{n_{\mathrm{H}}}{10^{-5} \mathrm{~cm}^{-3}}\right)^{4 / 3}\left(\frac{T}{10^{4} \mathrm{~K}}\right)^{-1 / 6} \\
& \times\left(\frac{J_{\mathrm{L}}}{10^{-21} \mathrm{erg} \mathrm{s}^{-1} \mathrm{~cm}^{-2} \mathrm{~Hz}^{-1} \mathrm{sr}^{-1}}\right)^{2 / 3},
\end{aligned}
$$

which is shown in Fig. 6 as solid lines. The indices in this formula can be derived analytically following Inoue and Kamaya (2004). From equations (A4) and (A7) in Inoue and Kamaya (2004), we find $\Gamma_{\text {pe }} \propto$ $J_{\mathrm{L}}^{2 /(p+\beta+1)} n_{\mathrm{H}}^{2-2 /(p+\beta+1)} T^{3 / 2-(2 p+2 \beta+1) /(p+\beta+1)}$, where $\beta$ is the emissivity (or absorption) index of the dust: $Q_{v} \propto v^{-\beta}$. Here, we have $p=1$ and $\beta \approx 1$, then, we obtain the indices in Eq. (16).

The deviation of the heating rates from the formula for $T=10^{5} \mathrm{~K}$ and $n_{\mathrm{H}}>2 \times 10^{-5} \mathrm{~cm}^{-3}$ is due to the relative significance of the cooling by the electron capture (see 


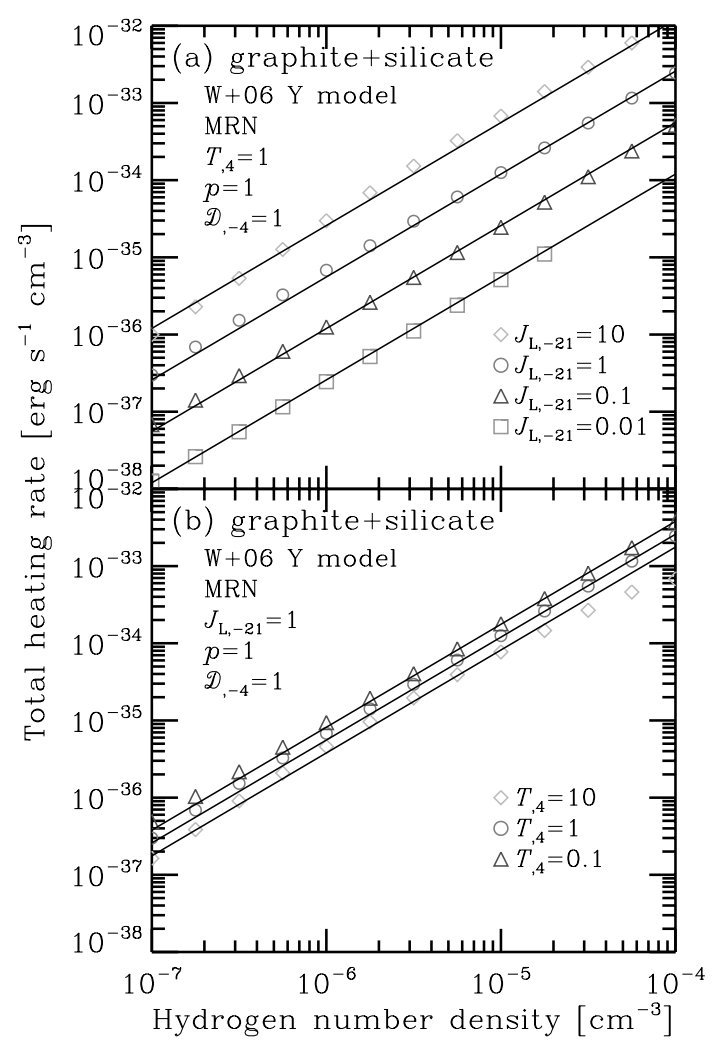

Fig. 6. Same as Fig. 4 but for various settings. The photoelectric yield model is the W+06 model. We assume that the dust consists of a mixture of graphite and silicate (50\% each in mass) with the MRN size distribution. (a) Different intensities at the Lyman limit of the radiation field: $J_{\mathrm{L}} / 10^{-21} \mathrm{erg} \mathrm{s}^{-1} \mathrm{~cm}^{-2} \mathrm{~Hz}^{-1} \mathrm{sr}^{-1}=10$ (diamonds), 1 (circles), 0.1 (triangles), and 0.01 (squares). (b) Different temperatures of the gas: $T / 10^{4} \mathrm{~K}=10$ (diamonds), 1 (circles), and 0.1 (triangles). Other assumed quantities are noted in the panels. See the caption of Fig. 4 for the notation. The solid lines are the simple formula shown in Eq. (16).

Eq. (6)). Indeed, we find that the mean energy of photoelectrons from small $(<0.01 \mu \mathrm{m})$ graphite grains is smaller than the mean kinetic energy of the $10^{5} \mathrm{~K}$ gas in the case of $n_{\mathrm{H}}=10^{-4} \mathrm{~cm}^{-3}$, consequently, the net heating rate per such a graphite grain is negative. Thus, we have a reduction of the total heating rate for $T=10^{5} \mathrm{~K}$ and $n_{\mathrm{H}}>2 \times 10^{-5}$ $\mathrm{cm}^{-3}$ as found in Fig. 6(b) although the heating rate by silicate grains is still positive.

The validity of the formula presented in Eq. (16) is ensured for $n_{\mathrm{H}}=10^{-7}-10^{-4} \mathrm{~cm}^{-3}, J_{\mathrm{L}}=10^{-23}-10^{-20} \mathrm{erg}$ $\mathrm{s}^{-1} \mathrm{~cm}^{-2} \mathrm{~Hz}^{-1} \mathrm{sr}^{-1}$, and $T=10^{3}-10^{5} \mathrm{~K}$ within a uncertainty of $30 \%$, except for $n_{\mathrm{H}}>2 \times 10^{-5} \mathrm{~cm}^{-3}$ with $J_{\mathrm{L}}=10^{-23} \mathrm{erg} \mathrm{s}^{-1} \mathrm{~cm}^{-2} \mathrm{~Hz}^{-1} \mathrm{sr}^{-1}$ or $T=10^{5} \mathrm{~K}$. Note that there may be a much larger uncertainty in the photoelectric yield model. If one likes another size distribution rather than the standard MRN, for example the BF05 model discussed in Section 3.3, the heating rate might be scaled by a factor found in Fig. 5 or the ratio of the typical sizes in Table 1.

\section{Discussion}

\subsection{Amount of the intergalactic dust}

Inoue and Kamaya $(2003,2004)$ discussed the effect of the photoelectric heating by the intergalactic dust on the thermal history of the IGM, obtaining an upper limit of the intergalactic dust amount. However, we have already seen that the $\mathrm{W}+06$ yield model results in a factor of 2-4 reduction of the photoelectric heating rate relative to the WD01 model which was adopted in Inoue and Kamaya (2003, 2004). We can conclude that the upper limits obtained from the IGM thermal history are raised by a few factor. Even in this case, the final limit obtained by Inoue and Kamaya (2004), which is that the intergalactic dust mass should be less than $10 \%$ of the metal mass produced in galaxies, is not affected because the limit was obtained mainly from the reddening measurements of $\mathrm{SNe}$ Ia at $z=0.5$, especially for $\sim 0.1 \mu \mathrm{m}$ size grains.

\subsection{Can grains cause an inverted temperature-density relation in the IGM?}

Bolton et al. (2008) recently suggest an inverted temperature-density relation in the low density IGM at $z=2-3$. The temperature in the low density IGM was previously thought to be proportional to the density positively (e.g., Hui and Gnedin, 1997). However, Bolton et al. (2008) examined carefully the probability distribution function (PDF) of the flux in QSOs' spectra through the Lyman $\alpha$ forest in the IGM and found that the observed PDF is explained better by the negatively proportional temperaturedensity relation; i.e., a lower density IGM is hotter. This needs a more efficient heating source for lower density IGM. Bolton et al. (2008) suggested a radiation transfer effect (e.g., Abel and Haehnelt, 1999) for the mechanism.

The intergalactic dust may contribute to the heating in the low-density IGM. As shown in Figs. 4 and 5, the importance of the dust photoelectric heating increases in lower density medium, which is plausible for the inverted temperaturedensity relation. For example, we expect a factor of $\sim 2$ larger heating rate by dust than HeII photoionization heating in a medium with $1 / 10$ of the mean density at $z=2$ for the MRN size distribution and 1\% dust-to-gas ratio of the Milky Way. Thus, the dust photoelectric heating may cause the inverted temperature-density relation observed in the low-density IGM at $z=2-3$. This point should be examined further by implementing the dust heating in a cosmological hydrodynamics simulation. For this, the formula presented in Eq. (16) will be useful.

\subsection{Photoelectric effect before the cosmic reionization}

Finally, we examine if the dust photoelectric heating is efficient in the IGM before the cosmic reionization. Because of the prominent Gunn-Peterson trough in QSOs' spectra (e.g., Fan et al., 2006), the cosmic reionization epoch should be at $z>6$. Here, we consider the IGM at $z \sim 10$.

Prior to the reionization, the ionizing background radiation does not exist although a nonionizing UV background can be established by primordial galaxies or active blackhole-accretion disk systems. An X-ray background radiation may also exist (e.g., Venkatesan et al., 2001). We consider two cases; one is the case with only a nonionizing UV background radiation and the other is the case with additional X-ray background radiation. For simplicity, we assume the background radiation to be a power-law with the spectral index $p=1$ and the intensity at the Lyman limit $J_{\mathrm{L}}=1 \times 10^{-21} \mathrm{erg} \mathrm{s}^{-1} \mathrm{~cm}^{-2} \mathrm{~Hz}^{-1} \mathrm{sr}^{-1}$. However, we assume no intensity between $E_{\mathrm{UV}}^{\max }=13.6 \mathrm{eV}$ and 
Table 2. Photoelectric heating in the early Universe.

\begin{tabular}{|c|c|}
\hline Common setting & \\
\hline$z$ & 10 \\
\hline$n_{\mathrm{H}}$ & $3 \times 10^{-4} \mathrm{~cm}^{-3}$ \\
\hline $\mathcal{D}$ & $10^{-4}$ \\
\hline size distribution & MRN \\
\hline$J_{\mathrm{L}}$ & $1 \times 10^{-21} \mathrm{erg} \mathrm{s}^{-1} \mathrm{~cm}^{-2} \mathrm{~Hz}^{-1} \mathrm{sr}^{-1}$ \\
\hline$p$ & 1 \\
\hline$E_{\mathrm{UV}}^{\max }$ & $13.6 \mathrm{eV}$ \\
\hline \multicolumn{2}{|c|}{ Nonionizing UV only } \\
\hline$T$ & $30 \mathrm{~K}$ \\
\hline$x_{\mathrm{e}}$ & $10^{-4}$ \\
\hline$\Gamma_{\mathrm{pe}}$ & $7 \times 10^{-36} \mathrm{erg} \mathrm{s}^{-1} \mathrm{~cm}^{-3}$ \\
\hline$t_{\mathrm{pe}}$ & $9 \times 10^{9} \mathrm{yr}$ \\
\hline \multicolumn{2}{|c|}{ With X-ray background } \\
\hline$E_{X}^{\min }$ & $300 \mathrm{eV}$ \\
\hline$T$ & $10^{4} \mathrm{~K}$ \\
\hline$x_{\mathrm{e}}$ & 0.3 \\
\hline$\Gamma_{\mathrm{pe}}$ & $2 \times 10^{-33} \mathrm{erg} \mathrm{s}^{-1} \mathrm{~cm}^{-3}$ \\
\hline$\Gamma_{\mathrm{pi}, \mathrm{X}}^{\mathrm{HI}}$ & $2 \times 10^{-30} \mathrm{erg} \mathrm{s}^{-1} \mathrm{~cm}^{-3}$ \\
\hline
\end{tabular}

$E_{\mathrm{X}}^{\min }=300 \mathrm{eV}$. Thus, in the nonionizing UV only case, we have the background radiation only below $E_{\mathrm{UV}}^{\max }=13.6 \mathrm{eV}$. In the case with the $\mathrm{X}$-ray background, we have radiation below $E_{\mathrm{UV}}^{\max }=13.6 \mathrm{eV}$ and above $E_{\mathrm{X}}^{\min }=300 \mathrm{eV}$. The dust-to-gas ratio in the IGM at $z \sim 10$ is of course unknown, but we assume 1\% dust-to-gas ratio of the Milky Way as an example, i.e., $\mathcal{D}=10^{-4}$. Note that the results obtained in the following discussions are linearly scaled by the value of $\mathcal{D}$. The mean hydrogen density in the Universe at $z \sim 10$ is $3 \times 10^{-4} \mathrm{~cm}^{-3}$. Table 2 is a summary of the assumed quantities and results obtained below.

In the nonionizing UV radiation-only case, there is no efficient heating mechanism for the whole of the Universe although primordial objects can heat up their surrounding gas locally. Thus, the temperature of the gas far away the sources is kept to be that of the cosmic background radiation at the epoch: $\sim 30 \mathrm{~K}$. The electron fraction $x_{\mathrm{e}}$, i.e., the number density of electron relative to that of hydrogen nucleus, is $\sim 10^{-4}$ in this low temperature IGM (Galli and Palla, 1998). The nonionizing UV photons still cause the photoelectric effect of grains. In the assumed setting, we have found that grains are positively charged and the dust photoelectric heating rate becomes $\Gamma_{\mathrm{pe}} \simeq 7 \times 10^{-36} \mathrm{erg}$ $\mathrm{s}^{-1} \mathrm{~cm}^{-3}$ for the MRN size distribution with a graphite and silicate mixture (50\% each in mass). We compare this heating rate with the gas thermal energy density: $U_{\text {gas }}=$ $(3 / 2) n_{\mathrm{H}} k_{\mathrm{B}} T$. The time-scale doubling the gas temperature with the photoelectric heating is given by $t_{\mathrm{pe}} \equiv U_{\mathrm{gas}} / \Gamma_{\mathrm{pe}} \simeq$ $9 \times 10^{9}$ years. Since the age of the Universe at $z=10$ is about $5 \times 10^{8}$ years, we conclude that the dust photoelectric heating is not very efficient in this case although it may be the strongest heating mechanism for the IGM.

In the case with the additional $\mathrm{X}$-ray background radiation, the IGM is partially ionized by the X-ray and the temperature becomes $\sim 10^{4} \mathrm{~K}$ (e.g., Venkatesan et al., 2001). If we assume the ionization equilibrium and optically thin for the X-ray, the electron fraction becomes $x_{\mathrm{e}} \simeq 0.3$ for the current setting of the X-ray background. In this medium, the grains are positively charged and the dust photoelectric heating rate becomes $\Gamma_{\mathrm{pe}} \simeq 2 \times 10^{-33} \mathrm{erg} \mathrm{s}^{-1} \mathrm{~cm}^{-3}$. We have assumed the MRN size distribution with a graphite and silicate mixture (50\% each in mass) again. However, the HI photoionization heating is much more efficient as $\Gamma_{\mathrm{pi}, \mathrm{X}}^{\mathrm{HI}} \simeq 2 \times 10^{-30} \mathrm{erg} \mathrm{s}^{-1} \mathrm{~cm}^{-3}$. Therefore, we again conclude that the dust photoelectric heating is negligible in the early Universe filled with an X-ray background radiation.

\section{Conclusion}

We have updated our calculations made in Inoue and Kamaya $(2003,2004)$ of the dust photoelectric heating in the IGM with the new model of the dust photoelectric yield by Weingartner et al. (2006). This new yield model takes into account the effect of the photon and electron transfer in a grain, the photoelectric emission from inner shells of grain constituent atoms, the Auger electron emission, and the secondary electron emission. A comparison with the previous yield model by Weingartner and Draine (2001) shows that the new yield is smaller than the old one for $\sim 100 \mathrm{eV}$ photons. This reduction of the yield is due to the photon/electron transfer effect and reduces the electric potential on grains and the heating rate significantly. For example, if we integrate over the grain size with the standard MRN distribution, the dust photoelectric heating rate with the new yield model is a factor of $2-4$ smaller than that with the old yield model. The photoelectric heating rate is more important in lower density medium. If the dust-to-gas ratio in the IGM is $1 \%$ of that in the Milky Way and the size distribution is the standard MRN model, the dust heating rate dominates the $\mathrm{HI}$ and HeII photoionization heating rates when the gas number density is less than $\sim 10^{-6} \mathrm{~cm}^{-3}$, even with the new yield model.

We have examined the effect of the size distribution function on the heating rate because the heating rate is inversely proportional to the typical grain size as found in Eq. (11). Bianchi and Ferrara (2005) suggested that the size of the intergalactic dust is larger than $\sim 0.1 \mu \mathrm{m}$ because smaller grains are destroyed by sputtering in the hot gas halo during the transport of grains from the parent galaxy to the IGM. In this case, the heating rate is reduced by a factor of $\sim 5$ relative to that with the standard MRN size distribution. The size distributions expected by the dust formation model in supernova ejecta are also examined. The heating rate with the size distribution of the grains just produced in the ejecta is very similar to that with the MRN distribution. In contrast, the heating rate with the size distribution of the grains processed by the reverse shock in the supernova remnant is a factor of $\sim 3$ smaller than that with the MRN model. The shock-processed grains have a larger size than the pristine ones because smaller grains are destroyed. On the other hand, if we put only small grains in the IGM, the heating rate increases significantly. Therefore, we conclude that the size distribution of grains in the IGM is an essential parameter for determining the dust heating efficiency. Even in the worst case considered here, the dust heating is expected to be the dominant heating mechanism in the IGM at $z=0$ if the dust-to-gas ratio in the IGM is $1 \%$ of that in the Milky Way.

Since the dust photoelectric heating rate with the new 
yield model is reduced by a factor of 2-4 relative to that with the old yield model, the upper limit on the amount of the intergalactic dust obtained by Inoue and Kamaya (2003, 2004) may be affected. Indeed, the limit based on the thermal history of the IGM should be raised by a factor of a few. However, their final upper limit is mainly obtained from the reddening measurements of $z=0.5$ supernovae Ia. Therefore, their conclusion would not be affected very much.

Bolton et al. (2008) suggested an inverted temperaturedensity relation in the lower density IGM at $z=2-3$ based on recent observations of the Lyman $\alpha$ forest in QSOs' spectra. To explain this interesting phenomenon, we need a heating mechanism more efficient in a lower density medium. The dust photoelectric heating has such a property. Indeed, the dust heating rate even with the new yield model is a factor of 2 larger than the HeII photoionization heating rate in a medium with a density of $1 / 10$ of the mean in the Universe at $z=2$ if the dust-to-gas ratio is $1 \%$ of that in the Milky Way. Thus, the possibility of the dust heating is worth examining more in detail. For this aim, the simple formula describing the dust photoelectric heating in the IGM presented in Eq. (16) will be very useful.

Finally, we have discussed the effect of the dust photoelectric heating in the early Universe. Prior to cosmic reionization, the ionizing background radiation is not established, but there may be nonionizing UV background and $\mathrm{X}$-ray background radiations. In the low temperature IGM only with a nonionizing UV background radiation, the dust photoelectric heating is not very efficient although it may be the strongest heating mechanism in the medium. In the partially ionized IGM with an X-ray background radiation, the HI photoionization heating rate is three orders of magnitude larger than the dust heating rate if the dust-to-gas ratio is $1 \%$ of that in the Milky Way. Therefore, we conclude that the dust photoelectric heating in the early Universe is not very important at least in the mean density environment.

Acknowledgments. We appreciate comments from the reviewers, B. T. Draine and M. M. Abbas, which improved the quality of this paper very much. We are grateful to the conveners of the session "Cosmic Dust" in the 5th annual meeting of the AsiaOceania Geosciences Society for organizing the interesting workshop. AKI is also grateful to all members of the Department of Physics, Nagoya University, especially the $\Omega$ Laboratory led by Tsutomu T. Takeuchi, for their hospitality during this work. AKI is supported by KAKENHI (the Grant-in-Aid for Young Scientists B: 19740108) by The Ministry of Education, Culture, Sports, Science and Technology (MEXT) of Japan.

\section{References}

Abbas, M. M. et al., Photoelectric emission measurements on the analogs of individual cosmic dust grains, Astrophys. J., 645, 324-336, 2006.

Abel, T. and M. G. Haehnelt, Radiative transfer effects during photoheating of the intergalactic medium, Astrophys. J. Lett., 520, L13-L16, 1999.

Aguirre, A. and Z. Haiman, Cosmological constant or intergalactic dust? constraints from the cosmic far-infrared background, Astrophys. J., 532, 28-36, 2000.

Aguirre, A., L. Hernquist, N. Katz, J. Gardner, and D. Weinberg, Enrichment of the intergalactic medium by radiation pressure-driven dust efflux, Astrophys. J. Lett., 556, L11-L14, 2001a.

Aguirre, A., L. Hernquist, J. Schaye, D. H. Weinberg, N. Katz, and J. Gardner, Metal enrichment of the intergalactic medium at $z=3$ by galactic winds, Astrophys. J., 560, 599-605, 2001 b.
Aguirre, A., L. Hernquist, J. Schaye, N. Katz, D. H. Weinberg, and J. Gardner, Metal enrichment of the intergalactic medium in cosmological simulations, Astrophys. J., 561, 521-549, 2001c.

Alton, P. B., J. I. Davies, and S. Bianchi, Dust outflows from starburst galaxies, M. N. R. A. S., 343, 51-63, 1999.

Bendo, G. J., et al., The spectral energy distribution of dust emission in the edge-on spiral galaxy NGC 4631 as seen with spitzer and the James Clerk Maxwell Telescope, Astrophys. J., 652, 283-305, 2006.

Bertoldi, F., C. L. Carilli, P. Cox, X. Fan, M. A. Strauss, A. Beelen, A. Omont, and R. Zylka, Dust emission from the most distant quasars, Astron. Astrophys., 406, L55-L58, 2003.

Bianchi, S. and A. Ferrara, Intergalactic medium metal enrichment through dust sputtering, M.N.R. A. S., 358, 379-396, 2005.

Bolton, J. S., M. Viel, T.-S. Kim, M. G. Haehnelt, and R. F. Carswell, Possible evidence for an inverted temperature-density relation in the intergalactic medium from the flux distribution of the Ly $\alpha$ forest, $M$. N. R. A. S., 386, 1131-1144, 2008.

Corasaniti, P. S., The impact of cosmic dust on supernova cosmology, $M$. N. R. A. S., 372, 191-198, 2006.

Draine, B. T., Photoelectric heating of interstellar gas, Astrophys. J. Suppl. Ser., 36, 595-619, 1978.

Draine, B. T., Evolution of interstellar dust, in The evolution of the interstellar medium, edited by L. Blitz, 193-205, Astronomical Society of the Pacific, San Francisco, 1990.

Draine, B. T., Scattering by interstellar dust grains. I. Optical and ultraviolet, Astrophys. J., 598, 1017-1025, 2003.

Draine, B. T. and E. E. Salpeter, Destruction mechanisms for interstellar dust, Astrophys. J., 231, 438-455, 1979.

Draine, B. T. and B. Sutin, Collisional charging of interstellar grains, Astrophys. J., 320, 803-817, 1987.

Dwek, E., The evolution of the elemental abundances in the gas and dust phases of the galaxy, Astrophys. J., 501, 645-665, 1998.

Elfgren, E., F.-X. Désert, and B. Guiderdoni, Dust distribution during reionization, Astron. Astrophys., 476, 1145-1150, 2007.

Fan, X., C. L. Carilli, and B. Keating, Observational constraints on cosmic reionization, Ann. Rev. Astron. Astrophys., 44, 415-462, 2006.

Ferrarotti, A. S. and H.-P. Gail, Composition and quantities of dust produced by AGB-stars and returned to the interstellar medium, Astron. Astrophys., 447, 553, 2006.

Ferrara, A., F. Ferrini, B. Barsella, and J. Franco, Evolution of dust grains through a hot gaseous halo, Astrophys. J., 381, 137-146, 1991.

Ferrara, A., M. Pettini, and Yu. A. Shchekinov, Mixing metals in the early Universe, M. N. R. A. S., 319, 539-548, 2000.

Fruchter, A., J. H. Krolik, and J. E. Rhoads, X-ray destruction of dust along the line of sight to $\gamma$-ray bursts, Astrophys. J., 563, 597-610, 2001.

Galli, D. and F. Palla, The chemistry of the early Universe, Astron. Astrophys., 335, 403-420, 1998.

Goobar, A., L. Bergström, and E. Mörtsell, Measuring the properties of extragalactic dust and implications for the Hubble diagram, Astron. Astrophys., 384, 1-10, 2002.

Gunn, J. E. and B. A. Peterson, On the density of neutral hydrogen in intergalactic space, Astrophys. J., 142, 1633-1641, 1965.

Haardt, F. and P. Madau, Radiative transfer in a clumpy universe. II. The ultraviolet extragalactic background, Astrophys. J., 461, 20-37, 1996.

Hirashita, H., T. Nozawa, T. Kozasa, T. T. Ishii, and T. T. Takeuchi, Extinction curves expected in young galaxies, M.N.R. A. S., 357, 1077-1087, 2005.

Hirashita, H., T. Nozawa, T. T. Takeuchi, and T. Kozasa, Extinction curves flattened by reverse shocks in supernovae, M. N. R. A. S., 384, 17251732, 2008.

Hui, L. and N. Y. Gnedin, Equation of state of the photoionized intergalactic medium, M. N. R. A. S., 292, 27-42, 1997.

Inoue, A. K. and H. Kamaya, Constraint on intergalactic dust from thermal history of intergalactic medium, M. N. R. A. S., 341, L7-L11, 2003.

Inoue, A. K. and H. Kamaya, Amount of intergalactic dust: constraints from distant supernovae and the thermal history of the intergalactic medium, M. N. R. A. S., 350, 729-744, 2004.

Jones, A. P., A. G. G. M. Tielens, and D. J. Hollenbach, Grain shattering in shocks: The interstellar grain size distribution, Astrophys. J., 469, 740764, 1996.

Loeb, A. and R. Barkana, The reionization of the universe by the first stars and quasars, Ann. Rev. Astron. Astrophys., 39, 19-66, 2001.

Mathis, J. S., W. Rumpl, and K. H. Nordsieck, The size distribution of interstellar grains, Astrophys. J., 217, 425-433, 1977.

Maiolino, R., R. Schneider, E. Oliva, S. Bianchi, A. Ferrara, F. Mannucci, M. Pedani, and M. Roca Sogorb, A supernova origin for dust in a high- 
redshift quasar, Nature, 431, 533-535, 2004.

Montier, L. A. and M. Giard, The importance of dust in cooling and heating the InterGalactic Medium, Astron. Astrophys., 417, 401-409, 2004.

Nath, B. B., S. K. Sethi, and Y. Shchekinov, Photoelectric heating for dust grains at high redshifts, M.N.R. A. S., 303, 1-14, 1999.

Nozawa, T., T. Kozasa, H. Umeda, K. Maeda, and K. Nomoto, Dust in the early universe: Dust formation in the ejecta of population III Supernovae, Astrophys. J., 598, 785-803, 2003.

Nozawa, T., T. Kozasa, A. Habe, E. Dwek, H. Umeda, N. Tominaga, K. Maeda, and K. Nomoto, Evolution of dust in primordial supernova remnants: Can dust grains formed in the ejecta survive and be injected into the early interstellar medium?, Astrophys. J., 598, 955-966, 2007.

Osterbrock, D. E. and G. J. Ferland, Astrophysics of Gaseous Nebulae and Active Galactic Nuclei Second Edition, University Science Books, SauSalito California, 2006.

Rho, J., T. Kozasa, W. T. Reach, J. D. Smith, L. Rudnick, T. DeLaney, J. A. Ennis, H. Gomez, and A. Tappe, Freshly formed dust in the Cassiopeia A supernova remnant as revealed by the Spitzer Space Telescope, Astrophys. J., 673, 271-282, 2008.

Scott, J., J. Bechtold, A. Dobrzycki, and V. P. Kulkarni, A uniform analysis of the Ly $\alpha$ forest at $z=0-5$. II. Measuring the mean intensity of the extragalactic ionizing background using the proximity effect, Astrophys. J. Suppl., 130, 67-89, 2000.

Spizer, L. Jr., The dynamics of the interstellar medium. II. Radiation pressure, Astrophys. J., 94, 232-244, 1941.
Venkatesan, A., M. L. Giroux, and J. M. Shull, Heating and ionization of the intergalactic medium by an early X-ray background, Astrophys. J., 563, 1-8, 2001.

Weingartner, J. and B. T. Draine, Photoelectric emission from interstellar dust: Grain charging and gas heating, Astrophys. J. Suppl., 134, 263281, 2001.

Weingartner, J., B. T. Draine, and D. K. Barr, Photoelectric emission from dust grains exposed to extreme ultraviolet and X-ray radiation, Astrophys. J., 645, 1188-1197, 2006.

Williams, B. J., et al., Dust destruction in fast shocks of core-collapse supernova remnants in the Large Magellanic Cloud, Astrophys. J. Lett., 652, L33-L56, 2006.

Xilouris, E., P. Alton, J. Alikakos, K. Xilouris, P. Boumis, and C. Goudis, Abundant dust found in intergalactic space, Astrophys. J. Lett., 651, L107-L110, 2006.

Yamada, K. and T. Kitayama, Infrared emission from intracluster dust grains and constraints on dust properties, Publ. Astron. Soc. Jpn., 57, 611-619, 2005.

Yun, M. S., P. T. P. Ho, and K. Y. Lo, A high-resolution image of atomic hydrogen in the M81 group of galaxies, Nature, 372, 530-532, 1994.

Zhang, P. and P. S. Corasaniti, Cosmic dust induced flux fluctuations: Bad and good aspects, Astrophys. J., 657, 71-75, 2007.

A. K. Inoue (e-mail: akinoue@las.osaka-sandai.ac.jp) and H. Kamaya 